\title{
Discrimination performance as affected by duration of shock for either the correct or incorrect response'
}

\author{
George J. Wischner and Harry Fowler \\ UNIVERSITY OF PITTSBURGH
}

\begin{abstract}
The present study extends our assessment of various shock parameters and training procedures as possible determinants of the paradoxical facilitating effect of shock for the correct response in discrimination training. Eighty hungry rats were trained with a non-correction procedure to make a light-dark discrimination for food under various durations of shock for either the right or wrong response. Trend analyses showed that, with greater durations of shock, errors decreased for the shock-wrong Ss but remained constant for the shockright Ss and did not depart significantly from the performance of no-shock controls. The data delimit any broad generalization that shock for the correct response facilitates discrimination performance.

\section{Problem}

The paradoxical finding reported by Muenzinger and his colleagues (e. g., Muenzinger, 1934; Muenzinger et al., 1938) that shock for the correct response facilitates discrimination performance appears generally accepted despite the results of more recent investigations which have failed to demonstrate the effect and point up the import of considering various classes of determining variables, including training procedures and shock parameters. In one of these studies (Wischner et al., 1963), investigation of the effect of shock intensity showed that, with a non-correction procedure, increasing intensities of shock reduced errors for shock-wrong (SW) groups but increased them for shock-right (SR) groups, all of the latter being inferior to no-shock (NS) controls. These results were interpreted as favoring an avoidance interpretation of the function of shock and as being opposed to any broad generalization that shock for the correct response facilitates performance.

The present study extends the previous one by assessing the effect of shock duration (with intensity held constant) on discrimination performance in a situation involving shock for either the correct or incorrect response, and a non-correction training procedure.

\section{Method}

Subjects were 80 naive male albino rats, about 100 days old at the start of training. The Ss were caged individually in the experimental room under controlled temperature and an artificially illuminated day-night cycle.

The apparatus was an enclosed T maze, the dimensions of which are reported elsewhere (Wischner et al., 1963). Each maze section was comprised of two L-shaped strips of sheet metal, each L serving as one wall and half of the floor. Together, the strips provided two $11 / 2$ in floor surfaces separated by a $3 / 4$ in gap. The discriminative stimuli were a dark alley and the illumination provided by a $10 \mathrm{w}$ bulb positioned behind the frosted Plexiglas end wall of a goal compartment. A matched impedance shock system consisting of a 60-cps AC source and a series resistance of .3 megohms was used to deliver shock to $\mathrm{S}$ when $\mathrm{S}$ interrupted in the appropriate arm an infra-red photoelectric beam crossing the arm at a point midway between the choice and goal sections. A manual priming feature of the circuit insured that $\mathrm{S}$ received only one shock on any trial. When shock was administered, its intensity was held constant at $60 \mathrm{v}$, as measured across the output of the transformer, and its duration set at either $.1, .2$, or $.4 \mathrm{sec}$.

The experimental procedure included both pre-training and training phases, the former serving to reduce position and brightness preferences. A week prior to pretraining, each Swas started and maintained thereafter on a daily diet of $12 \mathrm{~g}$ Purina Lab Checkers, with water available ad libitum. For pre-training, each $\mathrm{S}$ received 16 forced-choice, food-reinforced trials, administered 4 per day with an intertrial interval of $15 \mathrm{~min}$., and randomly distributed with the restriction that half of the trials were to the left and half to the right with half of each of these sets being to a lighted and half to a dark goal. Food reinforcement (P. J. Noyes sugar pellets, $4 \mathrm{~mm}, 45 \mathrm{mg}$ ) was liberally spread throughout a goal compartment during the first pre-training day and thereafter systematically reduced until, on the last pretraining day, $S$ received only 2 pellets per trial.

Initially, for the training phase, 56 Ss were randomly assigned to 7 equally numbered groups: 3 SR and 3 SW groups, one for each of the 3 shock duration intervals, and an NS control group. Subsequently, and for reasons noted below, 24 additional Ss were randomly assigned in equal number to the NS and 3 SR groups. During training, food reinforcement ( 2 pellets) was obtained only in the lighted goal, the right-left position of which was varied according to a pre-determined random schedule. Training entailed a free-choice, non-correction procedure. All Ss received 4 trials per day for the first 6 training days, the intertrial interval being $15 \mathrm{~min}$. , and thereafter 8 trials per day until $\mathrm{S}$ met a criterion of 15 correct responses out of 16 consecutive choices with the last 8 being correct, or until a total of 200 training trials had been administered. 


\section{Results and Discussion}

Group mean errors for the initial sample of $56 \mathrm{Ss}$ are presented in Fig. 1 in blocks of 40 trials over the 200 trials of the experiment. It is evident that, relative to the NS group, only the SW Ss showed superior performance throughout the course of training. Because of generally orderly progression of the performance curves, group mean errors over the entire 200 training trials were subjected to statistical analysis. These data are presented in Fig. 2 as a function of shock duration.

Analysis of variance of the data presented in Fig. 2 for the initial sample of Ss (n per group $=8$ ) showed that overall group differences were significant ( F, 4.21; df, $6 / 49 ; \mathrm{p}<.005$ ) and further that the diverging linear trends for the SR and SW conditions with increasing shock durations were also reliable (F, 13.78; df, 1/49; $\mathrm{p}<.001)$. However, separate trend analyses involving the NS group ( 0.0 sec. shock) and either the SR or SW groups alone showed that only the linear decrement in errors over increasing durations for the SW Ss was significant (F, 7.40; df, 1/28; $\mathrm{p}<.02)$; differences between the NS and SR groups and the trends within these data were all unreliable $(F<1)$.

Since significant linear trends were obtained in the intensity study (Wischner et al., 1963) for both the SR and SW conditions, the present NS and SR groups were replicated with an additional 6 Ss per group in order to determine whether the non-significant trend for the SR condition related in any way to sampling factors. These additional data, combined with the original data, are shown in Fig. 2 by the broad-line function. An analysis of variance of these combined data also failed to yield any significant group differences or trend components $(\mathrm{F}<1)$.

The findings of the present study, like those of the previous intensity study, argue against any facilitating effect of shock for the correct response; none of the SR duration groups showed any evidence of this effect.

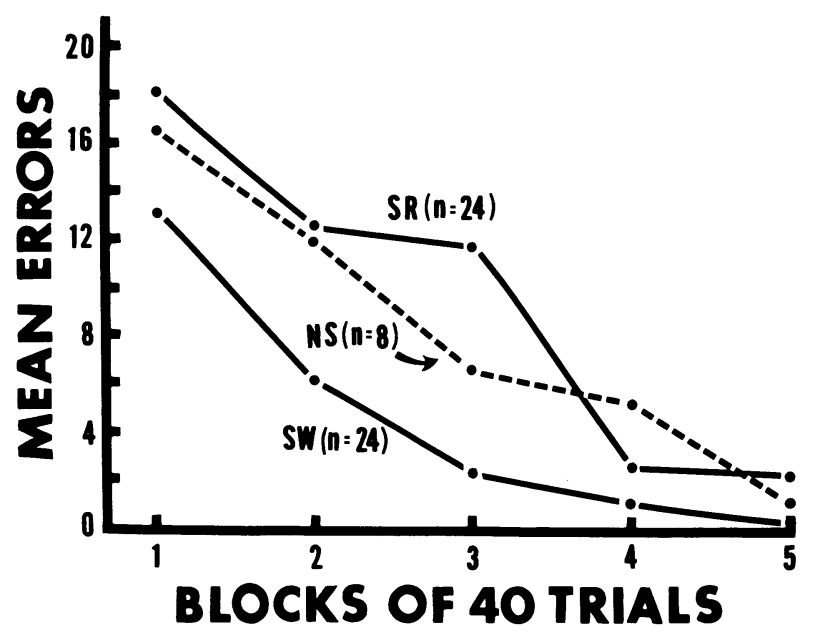

Fig. 1. Mean errors for the SR, SW and NS groups in blocks of 40 trials over the 200 trials of the experiment.

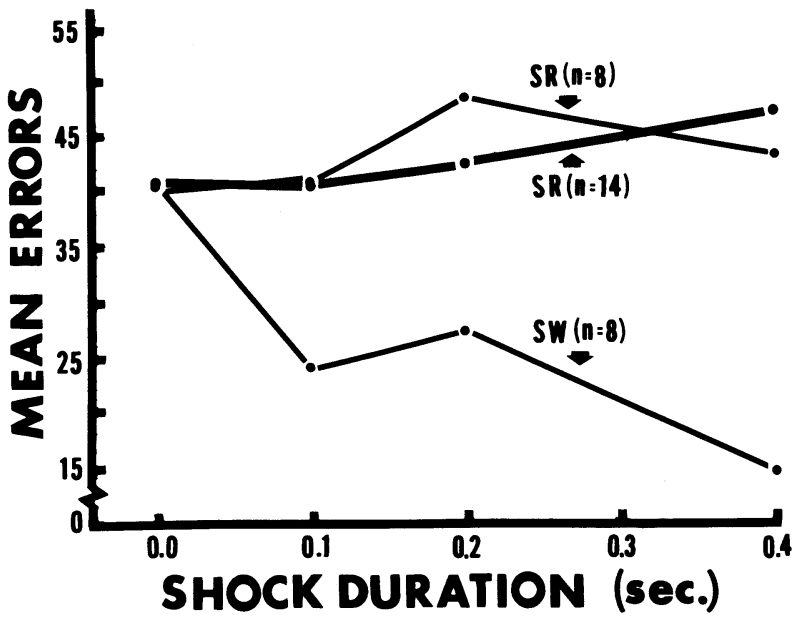

Fig. 2. Group mean errors over the 200 training trials as a function of shock duration.

Nevertheless, the failure to obtain an increment in errors with increasing shock durations for the SR condition remains to be explained. It might be argued that the maximum shock duration of $.4 \mathrm{sec}$. employed was too brief to be effective but, if this were the only important factor, a significant duration gradient would not be expected for the SW condition. A possible explanation of the data is to be found in accepting a dual function of shock. Through its aversive property, shock may produce avoidance of the cues with which it is associated, thereby increasing errors for SR Ss and decreasing them for SW Ss. However, as an additional c u e which is associated with either the correct or incorrect response, shock may also heighten the discriminability of the alternatives, thereby facilitating performance. For the SW condition, the avoidance cue functions should operate together to reduce errors, but with the SR condition these two functions may counteract one another, offsetting differences between NS and SR groups. Presumably with relatively high intensities of shock, as used in the previous study, the avoidance function of shock would predominate to produce the increasing SR error gradient obtained in that study.

\section{References}

MUENZINGER, K. F. Motivation in learning: I. Electric shock for correct responses in the visual discrimination habit. J.comp. Psychol, 1934, 17, 439-448.

MUENZINGER, K. F., BERNSTONE, A. H., \& RICHARDS, L. Motivation in learning: VIII. Equal amounts of electric shock for right and wrong responses in a visual discrimination habit. J.comp. Psychol., $1938,26,177-186$.

WISCHNER, G. J., FOWLER, H., \& KUSHNICK, S. A. Effect of strength of punishment for "correct" or "incorrect" responses on visual discrimination performance. J. exp. Psychol, 1963, 65, 131-138.

\section{Note}

1. This study was supported in part by Grants G-14312 from the National Science Foundation and $\mathrm{MH}-08482$ from the National Institutes of Health, United States Public Health Service. Grateful acknowledgment is made to Stephen A. Kushnick and Martin Samuels for their assistance in the conduct of this experiment. 